\title{
Targeting intermediary metabolism enhances the efficacy of BH3 mimetic therapy in hematologic malignancies
}

\author{
Aoula Al-Zebeeby, ${ }^{1}$ Meike Vogler, ${ }^{2}$ Mateus Milani, ${ }^{1}$ Caitlin Richards, ${ }^{1}$ Ahoud \\ Alotibi, ${ }^{1}$ Georgia Greaves, ${ }^{1}$ Martin J.S. Dyer, ${ }^{3}$ Gerald M. Cohen ${ }^{1,4}$ and Shankar \\ Varadarajan $^{1,4 *}$
}

Haematologica 2019

Volume 104(5):1016-1025

${ }^{1}$ Department of Molecular and Clinical Cancer Medicine, Institute of Translational Medicine, University of Liverpool, UK; ${ }^{2}$ Institute for Experimental Cancer Research in Pediatrics, Goethe-University, Frankfurt, Germany; ${ }^{3}$ Ernest and Helen Scott Haematological Research Institute, Leicester Cancer Research Centre, University of Leicester, Leicester Royal Infirmary, UK and ${ }^{4}$ Department of Molecular and Clinical Cancer Pharmacology, Institute of Translational Medicine, University of Liverpool, UK

\section{ABSTRACT}

B H3 mimetics are novel targeted drugs with remarkable specificity, potency and enormous potential to improve cancer therapy. However, acquired resistance is an emerging problem. We report the rapid development of resistance in chronic lymphocytic leukemia cells isolated from patients exposed to increasing doses of navitoclax (ABT-263), a BH3 mimetic. To mimic such rapid development of chemoresistance, we developed simple resistance models to three different BH3 mimetics, targeting BCL-2 (ABT-199), BCL-X $\mathrm{X}_{\mathrm{L}}$ (A-1331852) or MCL-1 (A-1210477), in relevant hematologic cancer cell lines. In these models, resistance could not be attributed to either consistent changes in expression levels of the anti-apoptotic proteins or interactions among different pro- and anti-apoptotic BCL-2 family members. Using genetic silencing, pharmacological inhibition and metabolic supplementation, we found that targeting glutamine uptake and its downstream signaling pathways, namely glutaminolysis, reductive carboxylation, lipogenesis, cholesterogenesis and mammalian target of rapamycin signaling resulted in marked sensitization of the chemoresistant cells to BH3 mimeticmediated apoptosis. Furthermore, our findings highlight the possibility of repurposing widely used drugs, such as statins, to target intermediary metabolism and improve the efficacy of $\mathrm{BH} 3$ mimetic therapy.

doi:10.3324/haematol.2018.204701

Check the online version for the most updated information on this article, online supplements, and information on authorship \& disclosures: www. haematologica.org/content/104/5/1016

(C)2019 Ferrata Storti Foundation

Material published in Haematologica is covered by copyright. All rights are reserved to the Ferrata Storti Foundation. Use of published material is allowed under the following terms and conditions:

https://creativecommons.org/licenses/by-nc/4.0/legalcode. Copies of published material are allowed for personal or internal use. Sharing published material for non-commercial purposes is subject to the following conditions:

https://creativecommons.org//icenses/by-nc/4.0/legalcode, sect. 3. Reproducing and sharing published material for commercial purposes is not allowed without permission in writing from the publisher.

\section{Introduction}

Failure to undergo apoptosis is a cardinal feature of cancer and several targeted therapies, such as the small molecule inhibitors targeting specific members of the anti-apoptotic BCL-2 family - navitoclax/ABT-263 (targeting BCL-2, BCL- $\mathrm{X}_{\mathrm{L}}$ and $B C L-w$ ) and venetoclax/ABT-199 (BCL-2 specific) - are aimed at facilitating cancer cell clearance by enhanced apoptosis. ${ }^{1-4}$ Recently, selective inhibitors of BCL-X $\mathrm{X}_{\mathrm{L}}$ (A1331852) and MCL-1 (A-1210477 and S63845) have also been synthesized. ${ }^{5.7}$ Despite their selectivity in targeting distinct anti-apoptotic BCL-2 family members, and remarkable potency in inducing rapid and extensive apoptosis in a wide variety of malignancies, resistance to $\mathrm{BH} 3$ mimetics, in particular venetoclax, is starting to be reported in the clinic. Elevated levels of multiple members of the anti-apoptotic BCL-2 family proteins, including BCL- $\mathrm{X}_{\mathrm{L}}$ and MCL-1, are often implicated in such chemoresistance..$^{8-13}$ Although it may be possible to target these proteins with a combination of selective $\mathrm{BH} 3$ mimetics, the potential toxicities associated with such combination therapy may be problematic.

Altered metabolism is a promising approach to enhance the efficacy of chemotherapeutic agents, as a requirement for intermediary metabolites, such as glucose and glutamine, for the survival and proliferation of cancer cells is well doc- 
umented.,14-19 This is a promising approach, as drugs targeting different stages of intermediary metabolism are already approved or in trials for treating different malignancies. ${ }^{20,21}$ In this study, we found a low level of resistance that developed in cells from patients with chronic lymphocytic leukemia (CLL) exposed to navitoclax. To mimic this modest resistance, we developed simple models of resistance to different $\mathrm{BH} 3$ mimetics and demonstrated that downregulating glutamine uptake or metabolism as well as its downstream signaling cascades, such as reductive carboxylation, lipogenesis and cholesterogenesis, result in enhanced apoptosis of cancer cells resistant to different $\mathrm{BH} 3$ mimetics, thus highlighting the possibility that inhibition of key regulatory enzymes of these metabolic pathways may enhance sensitivity to $\mathrm{BH} 3$ mimetic therapy.

\section{Methods}

\section{Reagents and antibodies}

ABT-263, A-1331852 and A-1210477 were from AbbVie (North Chicago, IL, USA), ABT-199, epigallocatechin gallate (EGCG), CB-839, simvastatin, rapamycin and torin-1 from Selleck Chemicals (Houston, TX, USA), gamma-L-glutamyl-pnitroanilide (GPNA) from Insight Biotechnology (Wembley, Middlesex, UK), azaserine from Cambridge Bioscience (Cambridge, UK), aminooxyacetate (AOA), sodium palmitate, dimethyl $\alpha$-ketoglutarate, oxaloacetate and citrate from SigmaAldrich (Gillingham, UK), L-glutamine from Life Technologies (Paisley, UK) and GSK2194069, SB204990, atorvastatin, pitavastatin and bafilomycin A1 from Tocris (Abingdon, UK). Antibodies against PARP, BCL-2, MCL-1, BAX, BAK and GAPDH were from Santa Cruz Biotechnology (Santa Cruz, CA, USA), caspase-3, caspase-9, BCL-X $\mathrm{L}, \mathrm{BCL}-\mathrm{w}, \mathrm{BIM}$, PUMA, BAD, IDH2, ACL, ACO2, ATG5 and ATG7 from Cell Signaling Technology (MA, USA), BID from Prof. J. Borst (The Netherlands Cancer Institute, Amsterdam, the Netherlands), NOXA from Millipore (Watford, UK) and SLC1A5, GLS, GFAT, GLUD1, IDH3, FASN and HMGR from Abcam (Cambridge, UK).

\section{Primary chronic lymphocytic leukemia cells and cell lines}

Peripheral blood samples from CLL patients were obtained with the patients' consent and ethics committee approval (06_Q2501_122) from Leicester Haematological Tissue Bank and cultured as described elsewhere. ${ }^{38}$ CLL samples were obtained from patients enrolled in a phase I/IIa study of ABT-263 (navitoclax) in patients with relapsed or refractory CLL (NCT00481091). Lymphocytes were purified and cultured in RPMI 1640 medium supplemented with 10\% fetal bovine serum (Life Technologies Inc.). Alternatively, blood from patients was incubated at $37^{\circ} \mathrm{C}$ in 48 -well plates and apoptosis assessed as described previously. ${ }^{39,40}$ Blood samples were collected prior to the first in vivo dose of navitoclax or $4 \mathrm{~h}$ after dosing during the lead-in period (day 1 of the lead-in period; L1D1), or day 1 of cycle 1 (C1D1), cycle 3 (C3D1) or cycle 5 (C5D1). Samples were collected $4 \mathrm{~h}$ after dosing as blood concentrations of ABT-263 were maximal at this time..$^{41}$ For culture of CLL cells, mouse fibroblast L cells were irradiated with 75 Gy and seeded in 24well plates $\left(3 \times 10^{5}\right.$ cells/well). CLL cells were cultured at $1.5 \times 10^{6}$ cells/well on the L cells and removed when required by gentle washing with RPMI before treatment. Mantle cell lymphoma (MAVER-1), chronic myeloid leukemia (K562) and multiple myeloma (NCI-H929) cell lines were cultured in RPMI 1640 medium but the medium was supplemented with $0.02 \% 2$-mercaptoethanol for culturing H929 cells. Cell lines were from either the Deutsche Sammlung von Mikroorganismen und Zellkulturen (DMSZ; Braunshweig, Germany) or the American Type Culture Collection (ATCC; Middlesex, UK) and subjected to short tandem repeat profiling to confirm their identity.

\section{Resistance models}

The different resistance models to relevant $\mathrm{BH} 3$ mimetics were developed by treating control cells (represented as A in all schemes) of MAVER-1, K562 and H929 to the relevant BH3 mimetics, ABT-199 (10 nM), A-1331852 (10 nM) or A-1210477 $(5 \mu \mathrm{M})$, respectively. In the first resistance model, cells were exposed to their appropriate BH3 mimetic for $24 \mathrm{~h}$ followed by 2 weeks without drug resulting in the cells depicted as B. These cells were then exposed to their appropriate $\mathrm{BH} 3$ mimetic for a further $24 \mathrm{~h}$ followed again by 2 weeks without drug resulting in C. This procedure was repeated twice more, resulting in E. In the second resistance model, cells were exposed to their appropriate $\mathrm{BH} 3$ mimetic for $24 \mathrm{~h}$ followed by 8 weeks without drug, resulting in the cells depicted as A4. The cells were collected every 2 weeks and labeled as A1, A2 and A3, respectively. In the third resistance model, cells were exposed to increasing concentrations of the appropriate $\mathrm{BH} 3$ mimetic every 5 days, resulting in cells depicted as A-a, A-b, A-c and A-d. The fourth model of resistance was made in a similar manner, but the 5-day treatment period was split into 2 days of treatment, followed by 3 days without drug, resulting in cells depicted as A-i, A-ii, A-iii and A-iv.

\section{Metabolic deprivation, supplementation and apoptosis measurements}

For glutamine deprivation experiments, cells were washed with phosphate-buffered saline and re-suspended in SILAC RPMI 1640 Flex Media (Life Technologies Inc.), supplemented with glucose $(2 \mathrm{mg} / \mathrm{mL})$ and $10 \%$ fetal bovine serum, for $16 \mathrm{~h}$. For supplementation studies, the indicated concentrations of metabolites were added to the glutamine-free media, immediately before glutamine deprivation. For lipid supplementation studies, sodium palmitate [dissolved in water at $70^{\circ} \mathrm{C}$ to form a stock concentration of $100 \mathrm{mM}$ and added dropwise into fatty acid-free bovine serum albumin (10\%) to produce a final concentration of $10 \mathrm{mM}$ ] was supplemented in the culture media. The extent of apoptosis in cells following different treatments was quantified by fluorescence activated cell sorting (FACS) after having stained the cells with annexin V-fluorescein isothiocyanate and propidium iodide to measure phosphatidylserine externalization, as previously described..$^{42}$

\section{Short interfering RNA knockdown, immunoprecipitation and western blotting}

Cells were transfected with $10 \mathrm{nM}$ of short interfering RNA (siRNA) against SLC1A5 (SI00079730), GLS (SI03155019), GFAT (SI03246355), GLUD1 (SI02654743), IDH2 (SI02654820), IDH3 (SI00300524), ACO2 (SI03019037), ACLY (SI02663332), FASN (SI00059752), HMGR (SI00017136), ATG5 (SI02633946) and ATG7 (SI04344830) from Qiagen Ltd. (Manchester, UK) using interferin (Polyplus Transfection Inc, NY, USA), according to the manufacturer's protocol and processed $72 \mathrm{~h}$ after transfection. Immunoprecipitation and western blotting were carried out according to standard protocols. ${ }^{43}$

\section{Statistical analysis}

One-way analysis of variance (ANOVA) multiple comparisons and the Fisher least significant difference test $(P \leq 0.01)$ were per- 
formed to compare sensitive and resistant cells. For samples from CLL patients, one-way repeated measures ANOVA with the Fisher least significant difference test $(P \leq 0.01)$ was used and statistics analyzed using GraphPad Prism 6 software (La Jolla, CA, USA).

\section{Results}

\section{Hematologic malignancies rapidly acquire resistance to BH3 mimetics}

The potential of $\mathrm{BH} 3$ mimetic therapy in cancer was first demonstrated in the treatment of BCL-2-dependent CLL using navitoclax/ABT-263. In a phase I/II clinical trial of navitoclax, CLL patients were treated for an initial lead-in period of 7 days with a low dose of navitoclax (100 mg daily) followed by five to seven cycles of treatment, with each cycle lasting 21 days during which the patients received $250 \mathrm{mg}$ navitoclax daily. Analysis of blood samples collected from these patients, either prior to the first in vivo dose of navitoclax or $4 \mathrm{~h}$ after dosing, during the different cycles of therapy revealed marked changes in the ability of navitoclax to induce apoptosis in the CLL cells (Figure 1A). The first in vivo dose of navitoclax on day 1 of the lead-in period (L1D1) resulted in a time-dependent induction of apoptosis, as assessed by phosphatidylserine externalization and ultrastructural changes, in comparison to that of CLL cells from the same patients prior to treatment (Figure 1A and Online Supplementary Figure S1). A progressive increase in resistance to navitoclax was observed in CLL cells in vivo during the different cycles of treatment (Figure 1A). Since these studies were carried out in whole blood, we wanted to ascertain whether the decrease in ABT-263-induced apoptosis in CLL cells could be attributed to chemoresistance. To test this, CLL cells were isolated from these patients at the beginning of each treatment cycle and exposed to increasing concentrations of ABT-263. A significant decrease [3-fold difference in the half maximal inhibitory concentration $\left(\mathrm{IC}_{50}\right)$ values between the lead-in period and cycle 5] was observed in their ability to undergo ABT-263-induced apoptosis (Figure 1B), demonstrating that continued dosing of patients with ABT-263 resulted in a modest, yet significant increase in chemoresistance.
A

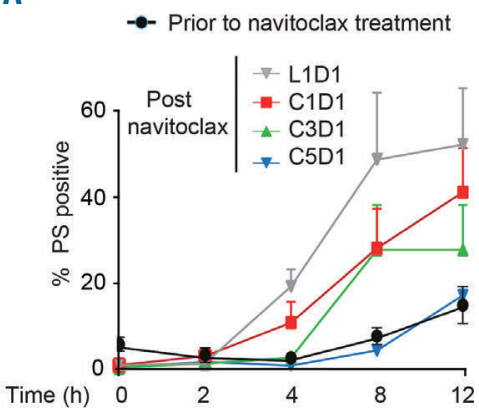

D

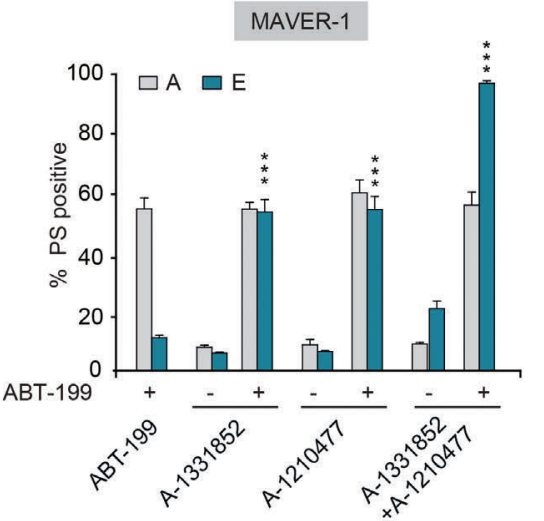

B

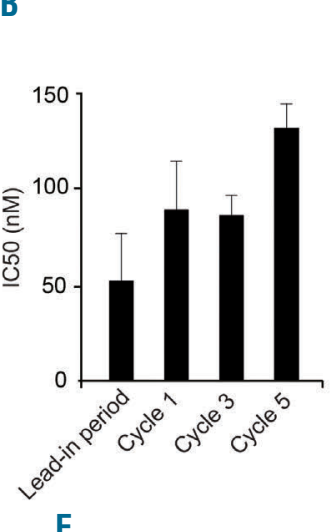

E

K562

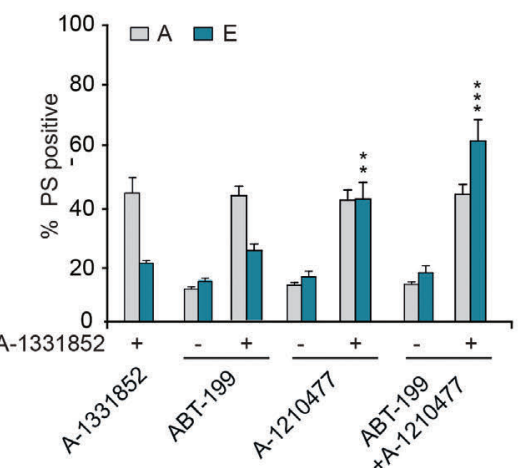

C

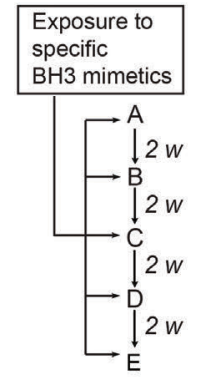

$\mathbf{F}$
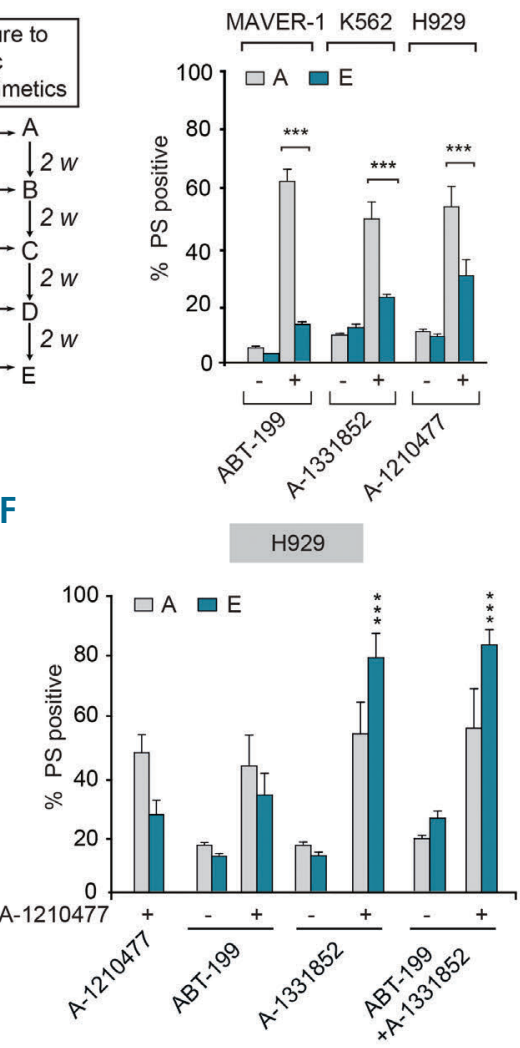

Figure 1. Hematologic malignancies rapidly acquire resistance to BH3 mimetics. (A) Blood samples collected from patients with chronic lymphocytic leukemia (CLL) $(n=5)$, either prior to the first in vivo dose of navitoclax or $4 \mathrm{~h}$ after dosing during different stages of treatment - day 1 of the initial lead-in-period (L1D1), day 1 of cycle 1 (C1D1), day 1 of cycle 3 (C3D1) or day 1 of cycle 5 (C5D1) - were incubated ex vivo and the extent of apoptosis in the CD19+ CLL cells was assessed at the indicated time points by measuring phosphatidylserine (PS) externalization. (B) CLL cells isolated from these patients at the beginning of each treatment cycle, as indicated in the figure, were exposed in vitro to increasing concentrations of ABT-263 and the extent of apoptosis was assessed: half maximal inhibitory concentration $\left(\mathrm{IC}_{50}\right)$ values are shown. (C) Scheme for establishing resistance to specific BH3 mimetics in relevant hematologic cell lines, as explained in the Methods section. Sensitive $[A]$ and resistant [E] cells of MAVER-1, K562 and H929 cell lines were exposed for $4 \mathrm{~h}$ to ABT-199 (10 nM), A-1331852 (10 nM) and A-1210477 (5 $\mu$ M), respectively, and apoptosis was assessed. (D-F) Combinations with some but not all BH3 mimetics restored apoptotic sensitivity of resistant [E] MAVER-1, K562 and H929 cells exposed for $4 \mathrm{~h}$ to ABT-199 $(10 \mathrm{nM}), \mathrm{A}-1331852(10 \mathrm{nM})$ or A-1210477 $(5 \mu \mathrm{M})$, respectively. $* * * P \leqslant 0.001, * \star P \leqslant 0.01$. Error bars $=$ mean \pm standard error of mean $(n=3)$. 
Since chemoresistance is an emerging problem in $\mathrm{BH} 3$ mimetic therapy, we extended these studies to more selective BH3 mimetics, such as ABT-199, which has replaced navitoclax owing to the dose-limiting thrombocytopenia associated with BCL- $\mathrm{X}_{\mathrm{L}}$ inhibition. ${ }^{3}$ Moreover, other selective $\mathrm{BH} 3$ mimetics that target $\mathrm{BCL}-\mathrm{X}_{\mathrm{L}}$ (A1331852) and MCL-1 (A-1210477 and S63845) have been introduced for use in several other malignancies..$^{57}$ Using these $\mathrm{BH} 3$ mimetics and relevant cancer cell lines, we tried to mimic the rapid resistance observed in CLL patients following navitoclax treatment (Figure 1A,B), in order to identify ways to tackle chemoresistance, as it emerges. For this, we chose the BCL-2-dependent MAVER-1, BCL-X-dependent K562 and MCL-1-dependent H929 cell lines and exposed them to ABT-199, A1331852 and A-1210477, respectively, to generate different models of resistance (Figure 1C and Online Supplementary Figure S2). Initial exposure of the relevant cell lines to the corresponding $\mathrm{BH} 3$ mimetic resulted in a rapid, time-dependent induction of apoptosis as assessed by the activation of caspase- 9 and caspase- 3 as well as cleavage of the canonical caspase substrate, PARP (Online Supplementary Figure S2A). Resistance to BH3 mimetics in these cells was generated by following the scheme presented in Figure 1C, when the initially sensitive cells [A] became relatively resistant [E], after four exposures (within 8 weeks) to their respective $\mathrm{BH} 3$ mimetic (Figure 1C). Similarly, a rapid resistance to the different $\mathrm{BH} 3$ mimetics was also observed using the other three resistance models (Online Supplementary Figure S2B-D). The rapid and modest resistance to the different $\mathrm{BH} 3$ mimetics in these cell lines was comparable to the extent of resistance observed in CLL cells during navitoclax therapy (Figure 1B).

\section{Resistance to BH3 mimetics can be overcome by inhibiting multiple BCL-2 family members}

Since resistance to $\mathrm{BH} 3$ mimetics has often been attributed to elevated expression levels of one or more antiapoptotic BCL-2 family members, we wanted to identify whether such changes could be responsible for the observed resistance. Comparison of the sensitive [A], intermediate $[\mathrm{C}]$ and resistant $[\mathrm{E}]$ cells from the different cell lines did not reveal any consistent differences in BCL2 family expression to explain the resistance (Online Supplementary Figure S3). We, therefore, sought to identify whether changes in protein-protein interactions among different pro-apoptotic BH3-only members and their antiapoptotic counterparts could explain the resistance to BH3 mimetics. To do this, we performed immunoprecipitation studies to isolate the anti-apoptotic proteins bound to BIM and PUMA, which were abundantly expressed in the three different cell types. However, in the sensitive [A] and resistant [E] MAVER-1 cells, immunoprecipitation of BIM and PUMA revealed similar binding of BCL-2 and BCL- $\mathrm{X}_{\mathrm{L}}$ and little or no binding to MCL-1 (Online Supplementary Figure S4). Likewise, no differences were observed in the binding of BIM and PUMA to BCL- $\mathrm{X}_{\mathrm{L}}$ and MCL-1 in sensitive and resistant K562 or H929 cells (Online Supplementary Figure S4).

Although the protein expression levels and immunoprecipitation studies did not support an involvement of other BCL-2 family proteins in the observed resistance, the resistance to ABT-199 observed in MAVER-1 cells was completely overcome by a combination of ABT-199 with either A-1331852 or A-1210477, but not by either A-
1331852 or A-1210477 alone, suggesting that the resistant cells depend not only on BCL-2 but also on BCL-X and/or MCL-1 for survival (Figure 1D). Furthermore, a combination of all three $\mathrm{BH} 3$ mimetics induced apoptosis in all the resistant cells, emphasizing the importance of all three anti-apoptotic BCL-2 family members in chemoresistance in these cells (Figure 1D). In K562 and H929 cells, the resistance was overcome by the combination of $\mathrm{A}$ 1331852 and A-1210477, but not ABT-199, thus implicating primary roles for $\mathrm{BCL}-\mathrm{X}_{\mathrm{L}}$ and $\mathrm{MCL}-1$ in chemoresistance (Figure 1E,F). Similar to the MAVER-1 cells, the chemoresistant K562 cells also exhibited enhanced apoptosis following treatment with a combination of all three BH3 mimetics (Figure 1E), suggesting that some contribution of BCL-2 could not be totally excluded in these cells. These observations were almost entirely reproducible in the other three models of resistance (Online Supplementary Figure S5), supporting the notion that BCL-X $\mathrm{X}_{\mathrm{L}}$ and/or MCL-1 contributed significantly to the observed chemoresistance in the different models.

\section{Modulation of glutamine uptake and/or metabolism enhances sensitivity to $\mathrm{BH} 3$ mimetics}

Although the above results demonstrate that a combination of $\mathrm{BH} 3$ mimetics can overcome resistance, such an approach targeting multiple members of the BCL-2 family requires careful evaluation of the therapeutic index, as these proteins perform redundant functions in the maintenance of normal cellular homeostasis. An alternative strategy to overcome chemoresistance to $\mathrm{BH} 3$ mimetics could be achieved by altered metabolism, as depriving cells of glutamine has recently been shown to overcome MCL-1-mediated chemoresistance in multiple myeloma. ${ }^{19}$ In our experiments, glutamine deprivation for $16 \mathrm{~h}$ alone did not exhibit any effect on overall cell survival and yet sensitized both the sensitive $[\mathrm{A}]$ and resistant $[\mathrm{E}]$ cells to BH3 mimetic-mediated apoptosis (Figure 2A). The increase in apoptosis observed in both sensitive and resistant cells indicates that glutamine deprivation most likely provides an additional cytotoxic cue that induces apoptosis in the sensitive and resistant cells, but could also bypass the resistance mechanism in the resistant cells. Nevertheless, our results suggest that targeting the glutamine metabolic pathway could enhance apoptosis and circumvent chemoresistance to $\mathrm{BH} 3$ mimetics in all our resistance models (Figure 2A and Online Supplementary Figure S6). To investigate the therapeutic potential of this approach, we wished to further understand how changes in glutamine metabolism might alter $\mathrm{BH} 3$ mimetic-mediated apoptosis.

Glutamine is transported into cells primarily via the SLC1A5 transporter and metabolized to glutamate, primarily via glutaminase (GLS)-mediated glutaminolysis. ${ }^{22}$ Alternatively, glutamate can be generated from glutamine as a by-product of the hexosamine biosynthetic pathway, during the conversion of fructose-6-phosphate to glucosamine-6-phosphate, catalyzed by the enzyme, glutamine:fructose-6-phosphate-amidotransferase (GFAT) (Figure 2B). ${ }^{22}$ Glutamate can then generate $\alpha$-ketoglutarate $(\alpha-K G)$ either via glutamate dehydrogenase (GLUD)-mediated oxidative deamination or a series of aminotransferase reactions (Figure 2B). ${ }^{14,22}$ Downregulation by RNA interference or pharmacological inhibition of key players involved in both glutamine uptake and its subsequent metabolism restored sensitivi- 
ty of chemoresistant K562 cells to A-1331852-mediated apoptosis, albeit to varying degrees (Figure 2C,D). While downregulation of SLC1A5 and GLS resulted in enhanced sensitivity to A-1331852-mediated apoptosis in the different cell lines tested, inhibition of other enzymes in the glutamine metabolic pathway produced more modest effects (Figure 2C,D and Online Supplementary Figure S7).

\section{Targeting reductive carboxylation enhances sensitivity to $\mathrm{BH} 3$ mimetics}

Metabolic supplementation of the glutamine-deprived cells with either glutamine or $\alpha-\mathrm{KG}$ restored the resistance of K562 cells to A1331852-induced apoptosis (Figure $3 \mathrm{~A})$. Since glutamine-derived $\alpha$-KG feeds into the tricarboxylic acid cycle, we explored the functions of this cycle and its intermediates in chemoresistance to $\mathrm{BH} 3$ mimetics. For this, we supplemented glutamine-deprived cells with tricarboxylic acid intermediates, such as oxaloacetate and citrate. Strikingly, supplementation with citrate, but not oxaloacetate, restored the resistance of K562 cells to A-1331852-induced apoptosis (Figure 3A). These results suggest that conversion of $\alpha$-KG to citrate via reductive carboxylation may play a role in regulating sensitivity to BH3 mimetics.

Reductive carboxylation involves the conversion of $\alpha$ KG to isocitrate (catalyzed by isocitrate dehydrogenases
1 and 2; IDH1/2), which then generates citrate (catalyzed by aconitase) (Figure $3 \mathrm{~B}$ ). ${ }^{23}$ While IDH1/2 catalyze reductive carboxylation of $\alpha-\mathrm{KG}$, another isoform of isocitrate dehydrogenase, IDH3, catalyzes the reverse conversion of isocitrate to $\alpha-K G .{ }^{23}$ Silencing the expression of IDH2 and aconitase, but not IDH3, restored the sensitivity of chemoresistant K562 cells to A-1331852-mediated apoptosis (Figure $3 \mathrm{C}, \mathrm{D}$ ), suggesting that the availability of citrate could be associated with the chemoresistance phenotype. To test this, IDH2-downregulated K562 cells were supplemented with citrate to identify whether addition of citrate could overcome the inhibition of reductive carboxylation and revert the associated increase in A1331852-induced apoptosis. Supplementation with citrate, but not glutamine or $\alpha-\mathrm{KG}$, did indeed restore the chemoresistance of IDH2-downregulated cells (Figure $3 E$ ), thus confirming the involvement of reductive carboxylation and the availability of citrate as crucial players in the observed chemoresistance.

\section{Downregulation of lipogenesis and cholesterogenesis enhances sensitivity to BH3 mimetics}

Since citrate generated as a consequence of reductive carboxylation of $\alpha-\mathrm{KG}$ is a major source of carbon for lipid synthesis, we investigated whether inhibition of lipogenesis could enhance sensitivity to $\mathrm{BH} 3$ mimetics
A

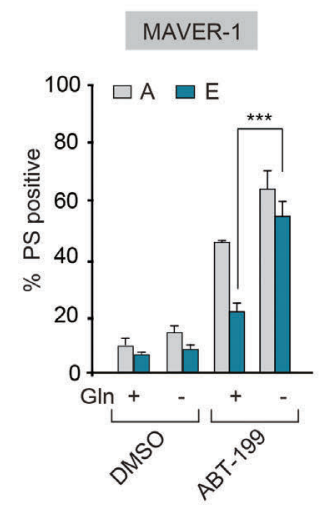

C
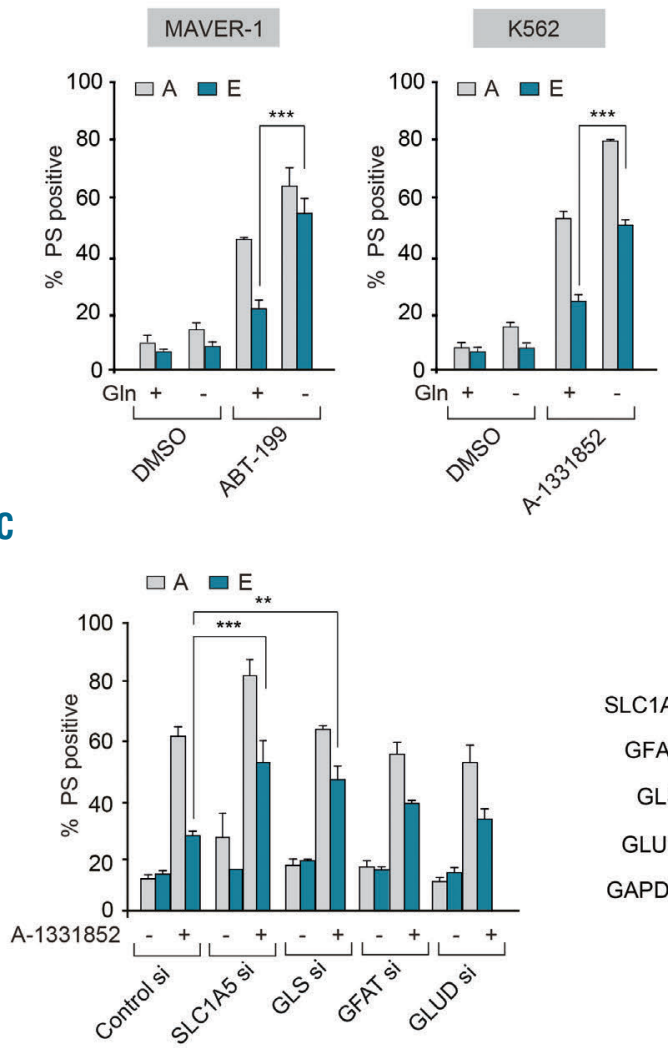

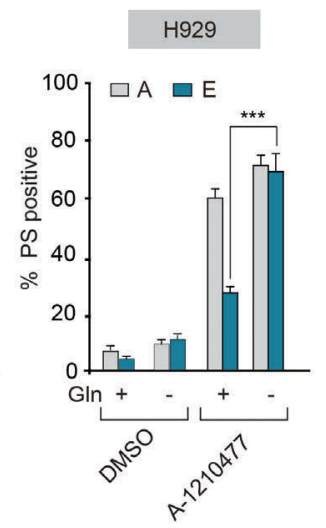

B

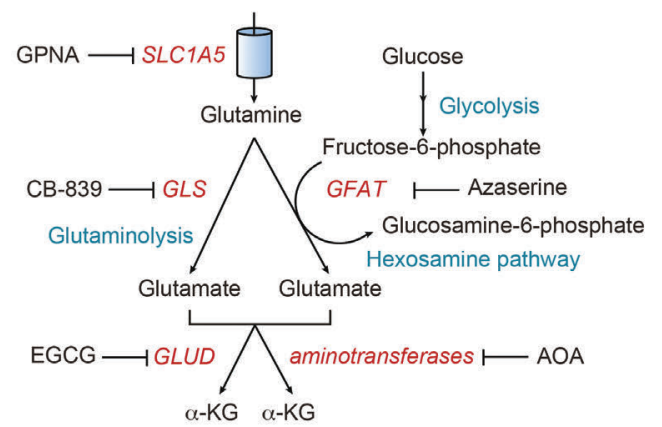

D

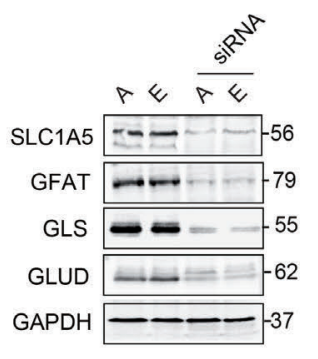

Figure 2. Inhibition of glutamine uptake and metabolism enhances sensitivity to BH3 mimetics. (A) Deprivation of glutamine (GIn) for $16 \mathrm{~h}$ restores the apoptotic sensitivity of resistant [E] MAVER-1, K562 and H929 cells to the indicated BH3 mimetic for $4 \mathrm{~h}$. (B) Scheme representing the pathway of glutamine uptake and metabolism. (C) Apoptotic sensitivity of K562 resistant [E] cells exposed to A-1331852 (10 nM) for $4 \mathrm{~h}$ was restored following genetic knockdown for $72 \mathrm{~h}$ with the indicated short interfering (si) RNA. (D) Apoptotic sensitivity of K562 resistant [E] cells exposed to A-1331852 (10 nM) for $4 \mathrm{~h}$ was restored following pharmacological inhibition of glutamine uptake or metabolism with GPNA $(5 \mathrm{mM})$ for $48 \mathrm{~h}$, CB-839 $(10 \mu \mathrm{M})$ for $72 \mathrm{~h}$, azaserine $(25 \mu \mathrm{M})$ for $16 \mathrm{~h}$ and AOA (500 $\mu \mathrm{M})$ for $24 \mathrm{~h}$ but not with EGCG $(50 \mu \mathrm{M})$ for $24 \mathrm{~h}$. Western blots confirmed the knockdown efficiency of the different siRNA. $* * * P \leqslant 0.001, * * P \leqslant 0.01$. Error bars $=$ mean \pm standard error of mean $(n=3)$. PS: phosphatidylserine; DMSO: dimethylsulfoxide. 
(Figure 4A). Using a complementary approach of genetic and pharmacological inhibition of ATP-citrate lyase (ACLY), which catalyzes the conversion of citrate to acetyl-CoA, ${ }^{24,25}$ as well as fatty acid synthase (FASN), which synthesizes long chain fatty acids following the condensation of acetyl-CoA and malonyl-CoA, ${ }^{26,27}$ we identified that modulation of the lipogenesis pathway, using either genetic silencing or pharmacological inhibition of ACLY (with SB204990) or FASN (with GSK2194069) could enhance sensitivity of cells to BH3 mimetics (Figure 4B,C and Online Supplementary Figure $S 7 C, D)$. Furthermore, metabolic supplementation with palmitate (the product of FASN (Figure 4A) in cells treated with GSK2194069 reverted the sensitized cells to their original chemoresistant phenotype (Figure 4D), thus obviating a requirement for FASN. These findings conclusively demonstrated that enhanced lipogenesis was associated with chemoresistance to $\mathrm{BH} 3$ mimetics and targeting lipogenesis could circumvent such resistance by enhancing $\mathrm{BH} 3$ mimetic-mediated apoptosis.

Acetyl-CoA generated from citrate can also feed into the cholesterol biosynthetic pathway, thus resulting in enhanced cholesterol production in cells. Targeting the rate-limiting step of cholesterol biosynthesis (catalyzed by HMG-CoA reductase; HMGR), either by genetic knockdown (Figure 4E) or pharmacological inhibition, using three widely used statins, simvastatin, atorvastatin and pitavastatin (Figure $4 \mathrm{~F}$ ), reversed resistance and restored the sensitivity of cells to $\mathrm{BH} 3$ mimetics (Figure 4E,F and Online Supplementary Figure S7E). Taken together, these data demonstrate that inhibition of several key players in lipid synthesis, including ACLY, FASN and HMGR, enhances the sensitivity to $\mathrm{BH} 3$ mimetics.

\section{Targeting the mammalian target of rapamycin signaling cascade enhances sensitivity to BH3 mimetics}

Since glutamine metabolism has been extensively implicated in mammalian target of rapamycin (mTOR) signaling, ${ }^{22,28}$ we speculated whether targeting mTOR kinases could enhance sensitivity to $\mathrm{BH} 3$ mimetics. Inhibition of mTOR kinases with rapamycin and torin-1 resulted in significant sensitization of cells to BH3-mediated apoptosis (Figure 5A and Online Supplementary Figure $S 7 F)$. To identify whether torin-1-mediated sensitization of cells to apoptosis was due to autophagy, we exposed the sensitive and resistant cells to bafilomycin A1 (Baf A1), which blocks autophagic flux by preventing lysosomal fusion of the autophagosomes. Exposure to
A

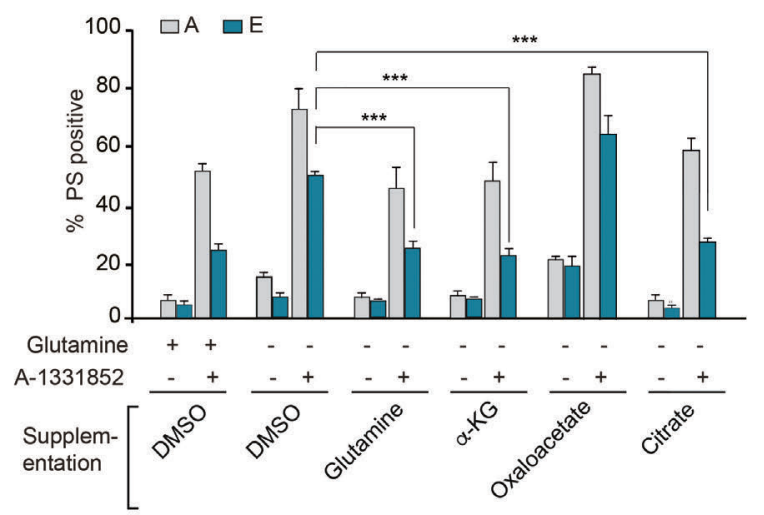

B

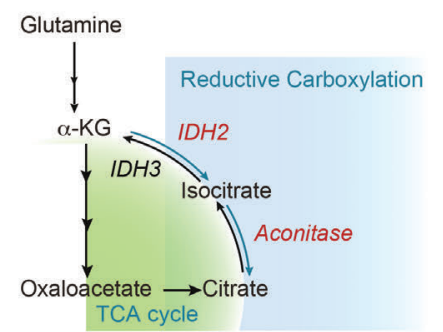

C

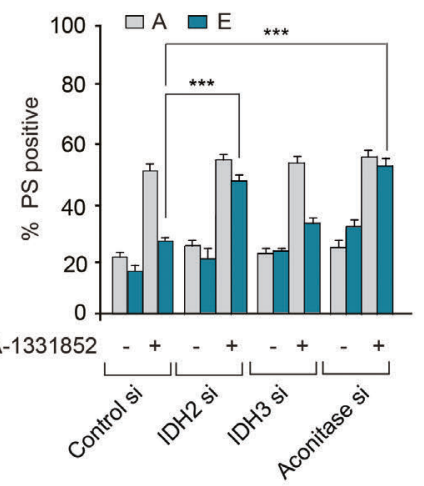

D

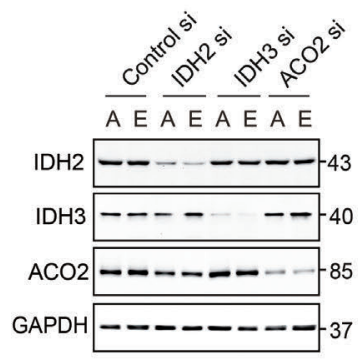

E

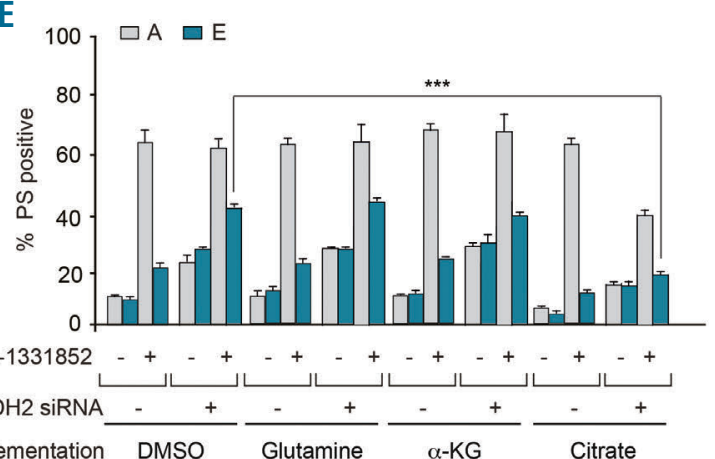

Figure 3. Modulation of reductive carboxylation enhances sensitivity to BH3 mimetics. (A) K562 sensitive [A] and resistant [E] cells were cultured in normal RPMI medium or glutamine-free medium with and without the supplementation of glutamine (2 mM), exposed to A-1331852 (10 nM) for $4 \mathrm{~h}$ and the extent of apoptosis assessed. Addition of citrate $(4 \mathrm{mM})$ and $\alpha$-ketoglutarate $(\alpha-K G)(4 \mathrm{mM})$ but not oxaloacetate $(4 \mathrm{mM})$ for $16 \mathrm{~h}$ reversed the sensitivity of the resistant [E] cells in glutamine-deprived media. (B) Scheme representing the link between the tricarboxylic acid (TCA) cycle and reductive carboxylation. (C) K562 sensitive [A] and resistant $[\mathrm{E}]$ cells were transfected with short interfering (si) RNA against IDH2, IDH3 and aconitase for $72 \mathrm{~h}$, followed by exposure for $4 \mathrm{~h}$ to A-1331852 and then apoptosis was assessed. (D) Western blots confirmed the knockdown efficiency of the different siRNA. (E) K562 [A] and [E] cells, transfected with a siRNA against IDH2 for 72 $\mathrm{h}$, were glutamine-deprived and then given or not supplementation with glutamine (2 mM), $\alpha$-ketoglutarate or citrate (both at $4 \mathrm{mM}$ ) for $16 \mathrm{~h}$ and the extent of apoptosis following exposure to A-1331852 $(10 \mathrm{nM})$ for $4 \mathrm{~h}$ was assessed. $* * * P \leqslant 0.001$. Error bars $=$ mean \pm standard error of mean ( $\mathrm{n}=3$ ). PS: phosphatidylserine; DMSO: dimethylsulfoxide. 
bafilomycin A1 failed to revert torin-1-mediated chemosensitization, suggesting that this effect could be independent of autophagy (Figure 5B). Furthermore, genetic silencing of autophagy proteins, ATG5 and ATG7, which are critical for the induction of autophagy, also failed to revert torin-1-mediated sensitization (Figure 5C), confirming our finding that mTOR inhibition circumvented resistance and enhanced sensitivity to BH3 mimetics independently of autophagy. In summary, our findings demonstrate that modulation of glutamine metabolism and its downstream signaling pathways, namely reductive carboxylation, lipogenesis and cholesterogenesis, as well as inhibition of mTOR signaling could enhance the therapeutic efficacy of BH3 mimetic therapy thereby circumventing chemoresistance to BH3 mimetics (Figure 5D).

\section{Targeting intermediary metabolism enhances} sensitivity to navitoclax in primary samples from patients with chronic lymphocytic leukemia

Our results indicate that targeting various facets of intermediary metabolism enhanced sensitivity to different BH3 mimetics in cell lines derived from relevant hematologic malignancies. To further extend our observa- tions in cell lines to primary samples from patients, we used CLL cells isolated from patients during the lead-in period (L1D1) as well as cells from the same patients after five cycles of navitoclax therapy (C5D1), as previously detailed in Figure 1. Using these samples, we wanted to determine whether modulating glutamine metabolism would enhance apoptosis mediated by navitoclax. For this, we exposed CLL cells to CB-839 and simvastatin for $24 \mathrm{~h}$ followed by navitoclax for $4 \mathrm{~h}$ and assessed the extent of apoptosis. In agreement with our cell line data, both CB-839 and statins overcame the resistance to navitoclax-mediated apoptosis in primary CLL cells (Figure 6), supporting the therapeutic translatability of our data from cell lines to patients.

\section{Discussion}

Anti-apoptotic BCL-2 family members are attractive drug targets both because of their high expression levels in several cancers and because of their well-characterized pro-survival roles. Even with extensive supportive in vitro data, the use of $\mathrm{BH} 3$ mimetics in treating cancer patients is still in its infancy, with venetoclax, a BCL-2-specific
A

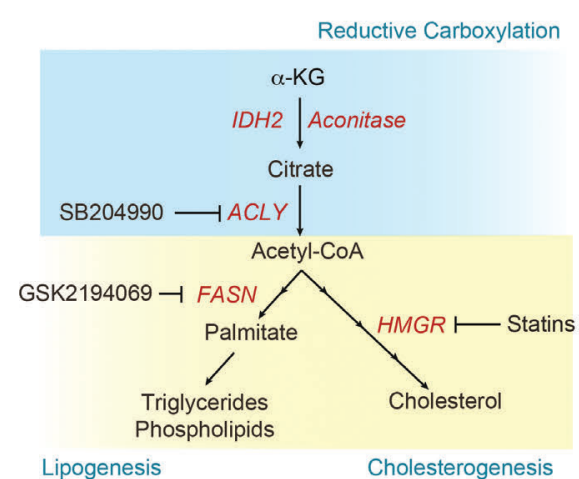

B

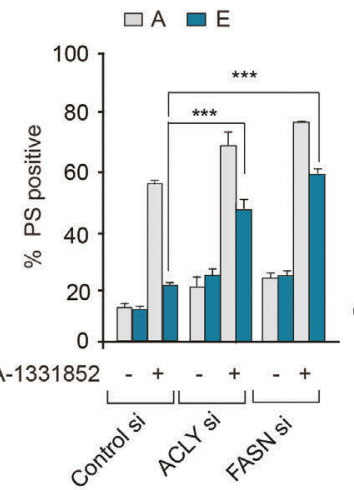

C
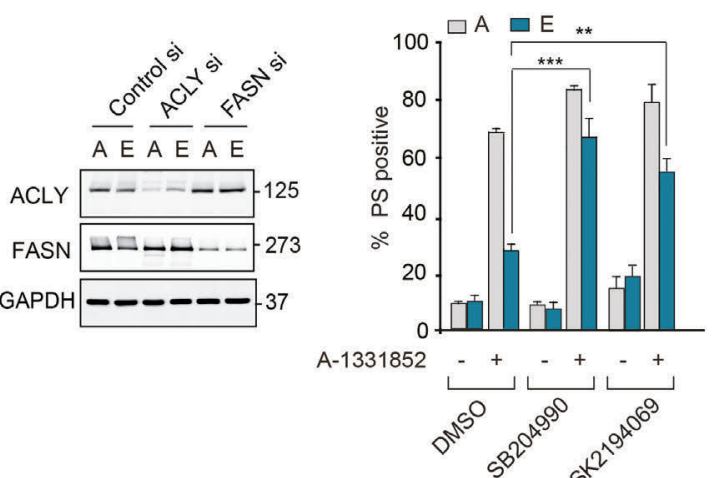

A-1331852

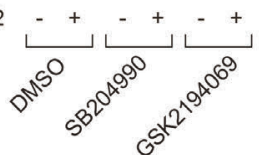

$\mathrm{F}$

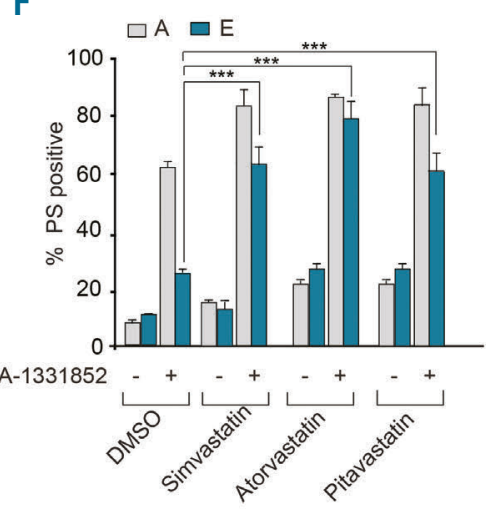

Figure 4. Inhibition of lipogenesis and cholesterogenesis enhances sensitivity to BH3 mimetics. (A) Scheme representing reductive carboxylation, lipogenesis and cholesterogenesis. (B) Apoptotic sensitivity of K562 resistant [E] cells exposed to A-1331852 (10 nM) for $4 \mathrm{~h}$ was restored following genetic knockdown for $72 \mathrm{~h}$ of key enzymes in fatty acid synthesis. Western blots confirmed the knockdown efficiency of the different short interfering (si) RNA. (C) Apoptotic sensitivity of K562 resistant [E] cells exposed to A-1331852 (10 nM) for $4 \mathrm{~h}$ was restored following pharmacological inhibition of key enzymes in fatty acid synthesis using SB204990 $(1 \mu \mathrm{M})$ for $72 \mathrm{~h}$ or GSK2194069 (100 nM) for $48 \mathrm{~h}$. (D) Metabolic supplementation of K562 sensitive [A] and resistant [E] cells with palmitate (50 $\mu \mathrm{M})$ for $48 \mathrm{~h}$ prior to the exposure of cells to GSK2194069 (100 nM) overcame the sensitizing effect of GSK2194069 on A-1331852-mediated apoptosis. (E) Genetic knockdown for $72 \mathrm{~h}$ of HMGR or (F) pharmacological inhibition of HMGR by simvastatin $(250 \mathrm{nM})$ for $72 \mathrm{~h}$, atorvastatin $(10 \mu \mathrm{M})$ for $48 \mathrm{~h}$ or pitavastatin $(1 \mu \mathrm{M})$ for $72 \mathrm{~h}$. $* * * P \leqslant 0.001$ $* * P \leqslant 0.01$. Error bars $=$ mean \pm standard error of mean $(n=3)$. PS: phosphatidylserine; DMSO: dimethylsulfoxide. 
A

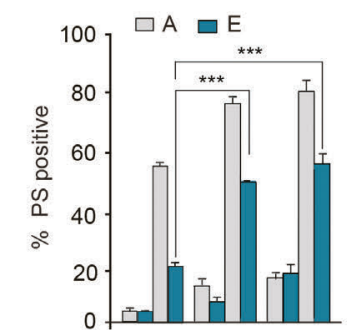

A-1331852

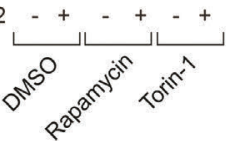

B

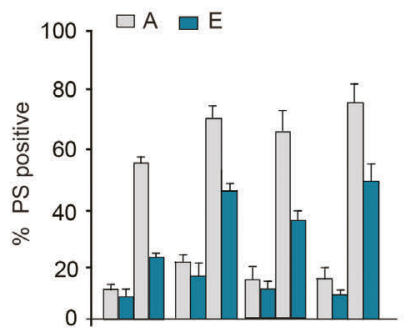

C

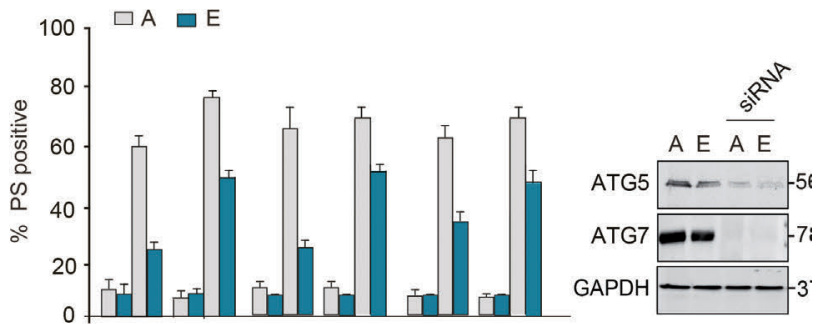

A-1331852

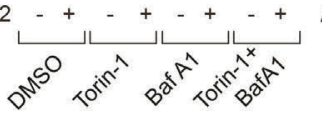

A-1331852

DMSO Torin-1 DMSO Torin-1 DMSO Torin-1

D

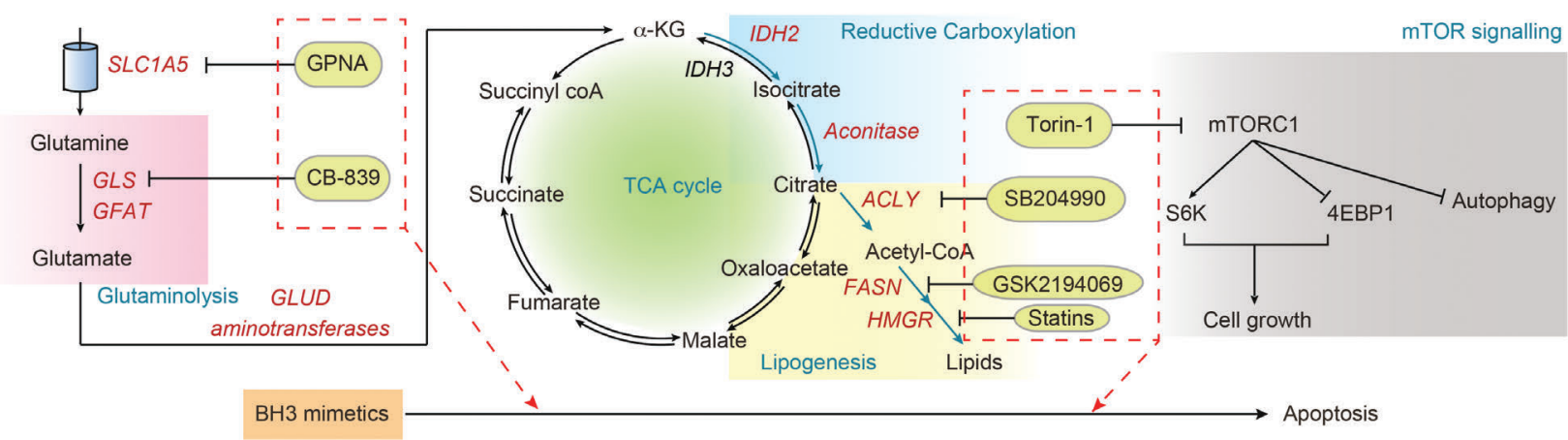

Figure 5. Modulation of mammalian target of rapamycin signaling enhances sensitivity to BH3 mimetics independently of autophagy. (A) Apoptotic sensitivity of $\mathrm{K} 562$ resistant $[\mathrm{E}]$ cells exposed to $\mathrm{A}-1331852(10 \mathrm{nM})$ for $4 \mathrm{~h}$ was restored following pharmacological inhibition of mTOR signaling using rapamycin (100 $\mathrm{nM}$ ) or torin-1 (10 nM) for $16 \mathrm{~h}$. (B) Inhibition of mTOR-regulated autophagy using 3-MA (10 mM) or bafilomycin A1 (100 nM) for $1 \mathrm{~h}$, followed by torin-1 (10 nM) for a further $16 \mathrm{~h}$, resulted in varying effects on A-1331852-mediated apoptosis. (C) Genetic knockdown of ATG5 and ATG7 for $72 \mathrm{~h}$ failed to revert torin-1 (10 nM)-mediated sensitization of apoptosis in K562 resistant [E] cells, following A-1331852 (10 nM) for $4 \mathrm{~h}$. Western blots confirmed the knockdown efficiency of ATG5 and ATG7 short interfering (si) RNA. $* * * P \leqslant 0.001$. Error bars $=$ mean \pm standard error of mean $(n=3)$. (D) Scheme representing glutamine uptake by SLC1A5 (inhibited by GPNA), glutaminolysis (inhibited by CB-839) to generate $\alpha$-ketoglutarate, reductive carboxylation of $\alpha$-ketoglutarate to generate citrate, which produces acetyl-CoA by a reaction catalyzed by ACLY (inhibited by SB204990), which eventually results in lipogenesis (inhibited by GSK2194069) and cholesterogenesis (inhibited by statins). Glutamine uptake, metabolism and its downstream signaling cascade can feed into mTOR signaling (inhibited by torin-1), all of which promote cell growth. In this study, we demonstrate that modulation of these distinct intermediary metabolic pathways could successfully sensitize cancer cells to BH3 mimetic-mediated apoptosis. PS: phosphatidylserine; DMSO: dimethylsulfoxide; TCA: tricarboxylic acid.

A

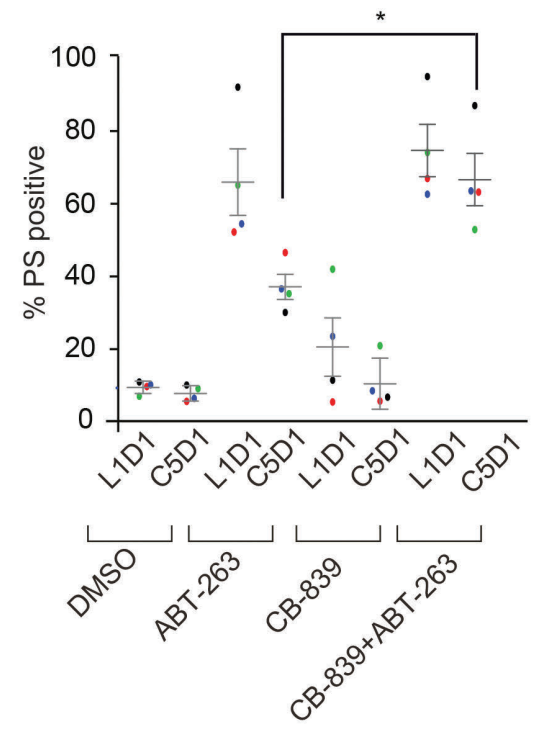

B

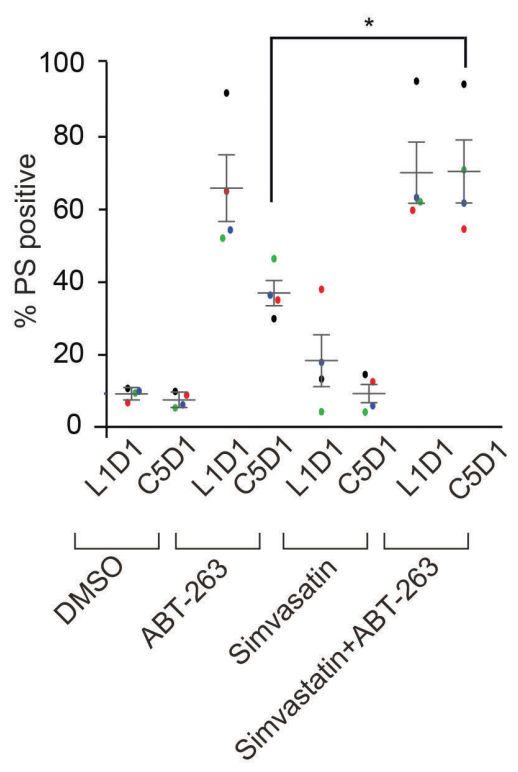

Figure 6. Inhibition of glutaminase and HMG-CoA reductase circumvents resistance to navitoclax-mediated apoptosis in primary chronic lymphocytic leukemia cells. Chronic lymphocytic leukemia cells isolated from five patients during the initial lead-in-period (L1D1) or day 1 of cycle 5 (C5D1) were cultured ex vivo on a feeder layer for $24 \mathrm{~h}$ and then exposed for a further $24 \mathrm{~h}$ to (A) CB-839 (50 $\mathrm{nM})$ or (B) simvastatin (10 $\mathrm{nM})$, and removed from the feeder layer for further exposure to navitoclax $(50 \mathrm{nM})$ for $4 \mathrm{~h}$. The extent of apoptosis was assessed as before. ${ }^{\star} P \leqslant 0.05$. Error bars $=$ mean \pm standard error of mean $(n=5)$. PS: phosphatidylserine; DMSO: dimethylsulfoxide. 
inhibitor, only recently having received approval for treatment of refractory CLL. ${ }^{4}$ The development of $\mathrm{BH} 3$ mimetics to target BCL- $\mathrm{X}_{\mathrm{L}}$ and MCL-1 in patients will be extremely valuable in the treatment of several types of cancer. However potential mechanisms of resistance to BH3 mimetics need to be recognized as they emerge and ways to circumvent resistance identified. Several resistance mechanisms, including mutations of the target site, ${ }^{29}$ post-translational modifications, ${ }^{30,31}$ and elevated levels of anti-apoptotic BCL-2 family members, ${ }^{8,11,32,33}$ have already been identified. While some of these resistance mechanisms could be overcome by co-administration of other specific $\mathrm{BH} 3$ mimetics that target $\mathrm{BCL}-\mathrm{X}_{\mathrm{L}}$ and/or MCL-1, ${ }^{5}$. 7 such inhibitors are not yet clinically available and the potential toxicities associated with the simultaneous inhibition of multiple BCL-2 family members are not known.

Attempts to identify measures that could overcome chemoresistance have led to exploration of the therapeutic potential of modulating intermediary metabolism in BH3 mimetic-mediated apoptosis. ${ }^{19,20,34}$ Although the mechanisms by which glutamine could regulate cancer cell proliferation have been extensively studied, the interrelationship between glutamine metabolism and apoptosis requires further study. It has been previously reported that glutamine-mediated apoptosis is dependent on $\mathrm{Myc}^{14}$ and that c-Myc activates glutaminolysis by upregulating both the glutamine transporter, SLC1A5, and glutaminase, GLS-1 ${ }^{35,36}$ However, we were unable to detect an increase in expression levels of Myc, SLC1A5 or GLS-1 in our resistance models (Figure 3 and data not shown). The ability of glutamine to regulate apoptosis and/or chemoresistance could also be due to its regulatory effect on mitochondrial oxidative phosphorylation. ${ }^{20}$
Although we do not entirely understand how glutamine metabolism impinges on apoptosis at this point, our data strongly support the notion that modulating glutamine metabolism and its related signaling pathways, such as reductive carboxylation, lipogenesis, cholesterogenesis and $\mathrm{mTOR}$ signaling, could enhance $\mathrm{BH} 3$ mimetic-mediated apoptosis in several hematologic malignancies (Figures 3-6). This is particularly promising, as glutaminase inhibitors, such as CB-839 and related drugs are already in clinical trials for the treatment of several malignancies ${ }^{20,37}$ and other drugs targeting cholesterogenesis, such as statins are the most commonly prescribed drugs to millions of people worldwide. While this manuscript was in preparation, an independent study comparing a large cohort of CLL patients, many of whom were statin users, found that response to venetoclax/ ABT-199 was enhanced among statin users in three different clinical trials. ${ }^{44}$ These findings highlight the possibility of repurposing several drugs targeting the intermediary metabolic pathways in conjunction with $\mathrm{BH} 3$ mimetic therapy to enhance therapeutic effectiveness and overcome the emerging chemoresistance in several cancers.

\section{Acknowledgments}

We thank AbbVie for inhibitors and Prof. J. Borst for antibodies. This work was supported by a NorthWest Cancer Research grant CR1040 (to SV and GMC), a studentship from the Ministry of Higher Education and Scientific Research and the University of Al-Qadisiyah, Iraq (fpr AA-Z), a Science Without Borders studentship, CNPq 233624/2014-7, from the Ministry of Education, Brazil (for MM) and a studentship from the Prince Sattam Bin Abdulaziz University, Saudi Arabia (for AA).

\section{References}

1 Hanahan D, Weinberg RA. Hallmarks of cancer: the next generation. Cell. 2011;144 (5):646-674

2 Tse C, Shoemaker AR, Adickes J, et al. ABT263: a potent and orally bioavailable $\mathrm{Bcl}-2$ family inhibitor. Cancer Res. 2008;68(9): 3421-3428.

3 Souers AJ, Leverson JD, Boghaert ER, et al. ABT-199, a potent and selective BCL-2 inhibitor, achieves antitumor activity while sparing platelets. Nat Med. 2013;19(2):202208.

4 Roberts AW, Davids MS, Pagel JM, et al. Targeting BCL2 with venetoclax in relapsed chronic lymphocytic leukemia. N Engl J Med. 2016;374(4):311-322.

5 Leverson JD, Phillips DC, Mitten MJ, et al. Exploiting selective BCL-2 family inhibitors to dissect cell survival dependencies and define improved strategies for cancer therapy. Sci Transl Med. 2015;7(279):279ra40.

6 Leverson JD, Zhang H, Chen J, et al. Potent and selective small-molecule MCL-1 inhibitors demonstrate on-target cancer cell killing activity as single agents and in combination with ABT-263 (navitoclax). Cell Death Dis. 2015;6:e1590.

7 Kotschy A, Szlavik Z, Murray J, et al. The MCL1 inhibitor S63845 is tolerable and effective in diverse cancer models. Nature. 2016;538(7626):477-482.
8 van Delft MF, Wei AH, Mason KD, et al. The $\mathrm{BH} 3$ mimetic ABT-737 targets selective $\mathrm{Bcl}-2$ proteins and efficiently induces apoptosis via Bak/Bax if Mcl-1 is neutralized. Cancer Cell. 2006;10(5):389-399.

9 Zhang H, Guttikonda S, Roberts L, et al. $\mathrm{Mcl}-1$ is critical for survival in a subgroup of non-small-cell lung cancer cell lines. Oncogene. 2010;30(16):1963-1968.

10 Gores GJ, Kaufmann SH. Selectively targeting Mcl-1 for the treatment of acute myelogenous leukemia and solid tumors. Genes Dev. 2012;26(4):305-311.

11 Vogler M, Butterworth M, Majid A, et al. Concurrent up-regulation of BCL-XL and BCL2A1 induces approximately 1000-fold resistance to ABT-737 in chronic lymphocytic leukemia. Blood. 2009;113(18):4403-4413.

12 Tahir SK, Smith ML, Hessler P, et al. Potential mechanisms of resistance to venetoclax and strategies to circumvent it. BMC Cancer. 2017;17(1):399.

13 Bose P, Grant S. Mcl-1 as a therapeutic target in acute myelogenous leukemia (AML). Leuk Res Rep. 2013;2(1):12-14.

14 Yuneva $M$, Zamboni N, Oefner $P$, Sachidanandam R, Lazebnik Y. Deficiency in glutamine but not glucose induces MYCdependent apoptosis in human cells. J Cell Biol. 2007;178(1):93-105

15 Wise DR, Thompson CB. Glutamine addiction: a new therapeutic target in cancer. Trends Biochem Sci. 2010;35(8):427-433.

16 Graham NA, Tahmasian M, Kohli B, et al.
Glucose deprivation activates a metabolic and signaling amplification loop leading to cell death. Mol Syst Biol. 2012;8:589.

17 Son J, Lyssiotis CA, Ying H, et al. Glutamine supports pancreatic cancer growth through a KRAS-regulated metabolic pathway. Nature. 2013;496(7443):101-105.

18 Still ER, Yuneva MO. Hopefully devoted to $\mathrm{Q}$ : targeting glutamine addiction in cancer. Br J Cancer. 2017;116(11):1375-1381.

19 Bajpai R, Matulis SM, Wei C, et al. Targeting glutamine metabolism in multiple myeloma enhances BIM binding to BCL-2 eliciting synthetic lethality to venetoclax. Oncogene. 2016;35(30):3955-3964.

20 Jacque N, Ronchetti AM, Larrue C, et al. Targeting glutaminolysis has antileukemic activity in acute myeloid leukemia and synergizes with BCL-2 inhibition. Blood. 2015;126(11):1346-1356.

21 Gross MI, Demo SD, Dennison JB, et al. Antitumor activity of the glutaminase inhibitor CB-839 in triple-negative breast cancer. Mol Cancer Ther. 2014;13(4):890-901.

22 Altman BJ, Stine ZE, Dang CV. From Krebs to clinic: glutamine metabolism to cancer therapy. Nat Rev Cancer. 2016;16(11):619634.

23 Al-Khallaf $\mathrm{H}$. Isocitrate dehydrogenases in physiology and cancer: biochemical and molecular insight. Cell Biosci. 2017;7:37.

24 Zaidi N, Swinnen JV, Smans K. ATP-citrate lyase: a key player in cancer metabolism. Cancer Res. 2012;72(15):3709-3714. 
25 Khwairakpam AD, Shyamananda MS, Sailo $\mathrm{BL}$, et al. ATP citrate lyase (ACLY): a promising target for cancer prevention and treatment. Curr Drug Targets. 2015;16(2):156163.

26 Menendez JA, Lupu R. Fatty acid synthase and the lipogenic phenotype in cancer pathogenesis. Nat Rev Cancer. 2007;7(10): 763-777.

27 Mashima T, Seimiya H, Tsuruo T. De novo fatty-acid synthesis and related pathways as molecular targets for cancer therapy. $\mathrm{Br} \mathrm{J}$ Cancer. 2009;100(9):1369-1372.

28 Saxton RA, Sabatini DM. mTOR signaling in growth, metabolism, and disease. Cell. 2017; 168(6):960-976.

29 Fresquet V, Rieger M, Carolis C, GarcíaBarchino MJ, Martinez-Climent JA. Acquired mutations in BCL2 family proteins conferring resistance to the $\mathrm{BH} 3$ mimetic ABT-199 in lymphoma. Blood. 2014;123(26): 4111-4119.

30 Konopleva M, Contractor R, Tsao T, et al. Mechanisms of apoptosis sensitivity and resistance to the $\mathrm{BH} 3$ mimetic ABT-737 in acute myeloid leukemia. Cancer Cell. 2006;10(5):375-388.

31 Mazumder S, Choudhary GS, Al-Harbi S, Almasan A. Mcl-1 phosphorylation defines ABT-737 resistance that can be overcome by increased NOXA expression in leukemic B cells. Cancer Res. 2012;72(12):3069-3079.
32 Chen S, Dai Y, Harada H, Dent P, Grant S. Mcl-1 down-regulation potentiates ABT-737 lethality by cooperatively inducing Bak activation and Bax translocation. Cancer Res. 2007;67(2):782-791

33 Lin $\mathrm{KH}$, Winter PS, Xie A, et al. Targeting MCL-1/BCL-XL forestalls the acquisition of resistance to ABT-199 in acute myeloid leukemia. Sci Rep. 2016;6:27696.

34 Chan SM, Thomas D, Corces-Zimmerman $\mathrm{MR}$, et al. Isocitrate dehydrogenase 1 and 2 mutations induce BCL-2 dependence in acute myeloid leukemia. Nat Med. 2015;21(2):178-184

35 Wise DR, DeBerardinis RJ, Mancuso A, et al. Myc regulates a transcriptional program that stimulates mitochondrial glutaminolysis and leads to glutamine addiction. Proc Natl Acad Sci U S A. 2008;105(48):18782-18787.

36 Liu W, Le A, Hancock C, et al. Reprogramming of proline and glutamine metabolism contributes to the proliferative and metabolic responses regulated by oncogenic transcription factor c-MYC. Proc Nat Acad Sci U S A. 2012:109(23):8983-8988.

37 Vander Heiden MG, DeBerardinis RJ. Understanding the intersections between metabolism and cancer biology. Cell. 2017;168(4):657-669.

38 Vogler M, Weber K, Dinsdale D, et al. Different forms of cell death induced by putative BCL2 inhibitors. Cell Death Differ.
2009:16(7):1030-1039.

39 Vogler M, Dinsdale D, Dyer MJS, Cohen GM. ABT-199 selectively inhibits BCL2 but not BCL2L1 and efficiently induces apoptosis of chronic lymphocytic leukaemic cells but not platelets. Br J Haematol. 2013; 163(7):139-142.

40 Vogler M, Furdas SD, Jung M, Kuwana T, Dyer MJS, Cohen GM. Diminished sensitivity of chronic lymphocytic leukemia cells to ABT-737 and ABT-263 due to albumin binding in blood. Clin Cancer Res. 2010;16(16): 4217-4225.

41 Roberts AW, Seymour JF, Brown JR, et al. Substantial susceptibility of chronic lymphocytic leukemia to BCL2 inhibition: results of a phase I study of navitoclax in patients with relapsed or refractory disease. J Clin Oncol. 2012;30(5):488-496.

42 Varadarajan S, Poornima P, Milani M, et al. Maritoclax and dinaciclib inhibit MCL-1 activity and induce apoptosis in both a MCL-1-dependent and -independent manner. Oncotarget. 2015;6(14):12668-12681.

43 Lucas CM, Milani M, Butterworth M, et al. High CIP2A levels correlate with an antiapoptotic phenotype that can be overcome by targeting BCL-XL in chronic myeloid leukemia. Leukemia. 2016;30(6):1273-1281.

44. Lee JS, Roberts A, Juarez D, et al. Statins enhance efficacy of venetoclax in blood cancers. Sci Transl Med. 2018;10(445). 